Indian Journal of Science and Technology

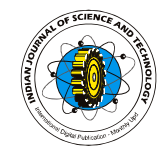

\title{
Comparison between alcoholic eucalyptus and nano-silver as a new nanocomposition in growth inhibition of Aspergilus niger
}

\author{
Nooshin Naghsh*, Mojgan Ghyasiyan, Safura Soleimani and Samira Torkan \\ Department of Biology, Falavarjan branch, Islamic Azad University, Falavarjan, Iran \\ *n_naghsh@yahoo.com, naghsh@iaufala.ac.ir
}

\begin{abstract}
Nano-silver particles are the integral aspect in functional nanotechnology filed. These nanoparticles have antibacterial and antifungal characteristics. Combination therapy is one of the new and specific methods for therapy of diseases in medicine and pharmacology fields. Interaction of alcoholic eucalyptus and nano-silver could be not performed in many investigations. For this reason, in this investigation, synergic effect of alcoholic eucalyptus and nano-silver in growth inhibition of Aspergilus niger were assayed A.niger were cultured in PDA medium. These fungi were treated with nanosilver and nano-silver -Eucalyptus ethanol extraction mixture in 12.5 and 50-ppm concentration. Then, Morphological changes and colony number in treatment and control groups were compared. Results of these investigations were shown that growth rate of $A$.niger decreased significantly $(\mathrm{P}<.01) 8$ day after treatment. In addition, growth and colony number of $A$.niger decreased significantly $(\mathrm{P}<0.01)$ in $12.5 \mathrm{ppm}$ concentration of nano-silver -Eucalyptus 24 day after treatment. These found could be suggested the therapy of human fungal diseases in medicine will be performed with mixture of nano-silver and eucalyptus ethanol extract. This nanomaterial could be used as antifungal agents. Specific investigations are necessary to improved that this combination has not any side effect in human. This nanomaterial could be suggested as a minor antifungal drug.
\end{abstract}

Keywords: Nano-silver, Nanobiotechnology, Eucalyptus ethanol extract, Aspergilus niger.

\section{Introduction}

One of greatest problems human faces today is how to combat against the microorganisms, which have gradually become resistant to chemical medicines. To overcome the situation, two approaches have been taken into consideration. The first is to increase the dosage of the medicine, but this approach has its own disadvantages, especially the enhanced risk from these chemical elements, created side effects, and their increased fatal effects on the environment (Moudgil \& Roberts, 2006). Taking pharmaceutical synthetic drugs irregularly and continuously results in an important phenomenon known as "autoimmunity" in bacteria and viruses. This phenomenon counteracts the effectiveness of drugs and finally leads to increase the dosage and to tend taking stronger medicines. The other disadvantage resulted from such medicines is that the increase in their side effects results in such diseases more severe and dangerous than the initial ones.

An innovative and new alternative is changes in formulation of antibacterial agents and synthetic drugs, that is, it can be used the combination therapy. Nowadays, medicinal herbs are consumed increasingly in the world because of exact knowledge of their antimicrobial effects. One of the most important, wellknown, and widespread used medicinal herbs is Eucalyptus because of whose biological activities such as antioxidant, antibacterial, and antiviral activities is used in traditional medicine. Eucalyptus, of the family Myrtaceae, is a tall tree with hard and durable wood. The primitive natives of Australia used the lives of Eucalyptus to cure the injuries and to alloy the fever. Bluish gum Eucalyptus or Australian fever tree is the most well known species with medical applications. Eucalyptus leaves have eucalyptol (Paux et al., 2004), Terpene alcohols, aliphatic aldehydes, isoamyl alcohols and Some of such compounds have various biological effects including antioxidant, antibacterial, and antifungal effects (Nakagawa et al., 1999). Considering the removed borders between the various sciences at present, it is undeniable and inevitable to use the nanotechnology in medical sciences and pharmacology.

Nanotechnology, as one of instances in sciences and technology developments, has gained many applications in human health and therapy. It has been taken into consideration the increasing application of nano sized metals as antimicrobial agent in new nanotechnology. One of dramatically scientific consequences of nanotechnology is nano-silver particles following carbon nano tubes in terms of widespread applications, which involve in many antimicrobial usages and in other applications in various fields such as medicine, agriculture, animal husbandry, home appliances, health and cosmetics (Arikan et al., 2001; Moudgil \& Roberts, 2006 ). In nano-silver technology, silver ions are in colloidal form in a suspension solution. The silver ions have more contact surface with the external space and so more effect on the environment due to their nano-size (Tahan et al., 2006). In general, the nano-particles, especially silver in nano-size, have changed physicochemical properties including size, shape, and surface area. All of such changes compared to the usual metal result in new considerable properties for nanoparticles, and in the case of nano-silver particles, it results in the increase in antimicrobial effect. Considering 
the fact that combining drugs may lead to synergic effects and the increase in the treatment rate of diseases.

Therefore, the present project compares the antibacterial effects of alcoholic extract of eucalyptus with the nano-silver particles on fungus - Aspergillus niger. This fungus was selected because it is the cause of disease Externa Otitis in human. Externa Otitis is the inflammation of external ear including auricle and ear canal, and is a very painful disease. In addition, the above fungi is the major cause of intestinal infections. Examining the special effects of nano-silver particles and of alcoholic extract of eucalyptus leaves in biological inhibition of A.niger is one of typical applications of new and innovative nanotechnology in biotechnology (Arikan \& Rex, 2000).

\section{Materials and methods}

In order to prepare the original sample, a vial of Aspergillus niger was purchased from Tehran Pars Institute. The professional medium PDA (Potato Dextrose Agar) was used to culture Aspergillus niger. The method is so that $39 \mathrm{~g}$ of PAD powder was weighted exactly, then this amount was reached to the desired volume through being diluted by two times distillated water in $1000 \mathrm{ml}$ volumetric flask. In order to sterilize the materials, the medium was placed in the autoclave with the temperature $121^{\circ} \mathrm{C}$ for $15 \mathrm{~min}$. at 15 bars. Then, the mediums were placed in filter-capped Erlenmeyer flask at the room temperature in order to be cooled. Each disposable plate was added by $25 \mathrm{ml}$ of medium near flame. To inoculate the medium with the fungus, a loop was sterilized by heating through the flame and after cooling the loop, the fungus was added to the medium by the loop (Christoforidis et al., 2006).

Nano-silver particles with $4.5 \mathrm{~nm}$ in mean diameters used in the study were purchased from Pars Nano Nasb Company in Tehran (Iran). Then, nano-silver particles were added to the medium in concentrations 50,100 , and $150 \mathrm{ppm}$. A control group was prepared by adding two times distilled water to one of the plats. It was recorded the morphologic changes, diameters, and number of colonies in treated groups in the $6^{\text {th }}, 7^{\text {th }}$, and $8^{\text {th }}$ days and then they were compared with the control group. In addition, it was calculated the rate of MIC of nano-silver particles.

\section{Determining MIC rate of nano-silver particles}

At first, a serial concentration of nano-silver particles solution is prepared starting from the concentration of 150 $\mathrm{ppm}$ in 6 test tubes. The method is that $2 \mathrm{~mL}$ of nanosilver solution with the concentration of $150 \mathrm{ppm}$ was added to the first test tube. The following dilutions were done by PDB medium. It was added $1 \mathrm{ml}$ of solution from the first tube and $1 \mathrm{cc}$ of PDB medium to the second test tube, so half due to the first test tube divide the concentration of the second test tube. This is repeated for the following test tubes, and $1 \mathrm{cc}$ of solution is removed from the last $\left(6^{\text {th }}\right)$ test tube, so all the test tubes contain 1 cc. of solution. Thus, the serial concentrations are 150,
$75,37.5,18.25,9.12$, and $4.56 \mathrm{ppm}$. Then, the fungus is sampled, that is, one loop of the spores from the medium is added to the PDB medium. The amount of medium does not matter, because the main purpose is the number of spores and to make them to reach the desired number. The number of spore is counted by the neobar slid. In order to facilitate the next calculations, the number of spores should be 1000. The number of needed spores for the next phase is 100 . In this phase, some of the solutioncontaining spore is added to the serial concentrations so that the number of spores is reached to 100 spores in each test tube. According to the calculations in order to reach the number of 100 spores in each test tube, it should be added $100 \mu \mathrm{l}$ of solution containing spores to every cc. of serial concentrations. The number 100 spores in each test tube is an optional number, and there is no purpose behind it. To facilitate the examination of the number of created colonies, it is added to the PDA medium of the prepared concentrations containing Aspergillus niger spores. The spores were counted in the first, second, third, $24^{\text {th }}$, and $48^{\text {th }}$ hour.

\section{Statistical test}

The needed statistical tests were done on the results using the software SIGMASTAT ${ }^{\mathrm{TM}}$ (Jandel Software, San Raphael, CA). In order to compare the diameter and the number of colonies in the treated groups with the ones in the control group, the mean comparison test was done by the t-test statistical method. The meaningfulness of differences between the samples is limited to $1 \%$ and $5 \%$. The plan used in this project is completely random. The samples for fungi were repeated 24 times. The t-test was done to compare the means. The software Excel plotted the software SPSS was done to measure the data statistically, and the graphs.

\section{Results and discussions}

For $A$.niger, the initial spores were counted as 100 by using the neobar slid. Figure 1 shows the inhibitory effects of nano-silver particles in concentration of $50 \mathrm{ppm}$ on the diameter of Aspergillus colony. The experimental findings showed that the least needed time for nano-silver particles to be effective is 6 days after treating. The inhibitory effect of nano-silver particles in $50 \mathrm{ppm}$ concentration on the diameter of fungi colonies showed meaningful reduction after 8 days of treatment $(P=0.02)$ (Fig.1), so that the mean number of colony in control group reached from 200 to $65 \pm 8$ in nano-silver group, $11 \pm 0.7$ in Eucalyptus group and $5 \pm 0.8$ in nano-silver and eucalyptus group. In 12.5-ppm concentration, the mean colony number in control group changed from 200 to $162 \pm 18$ in nano-silver group, $52 \pm 17$ in Eucalyptus group and $23 \pm 10$ in nano-silver and eucalyptus group (Fig.2). Comparison between fungi colony number in $12.5 \mathrm{ppm}$ concentration of nano-silver at different time of post treatment in A.niger were shown that 8 day post treatment is optimum time for inhibitory effects on nanosilver (Fig.3). 
Fig. 1. Comparison between fungi colony number of control and treatment groups in 50ppmm concentration of nanosilver 8-day post treatment in

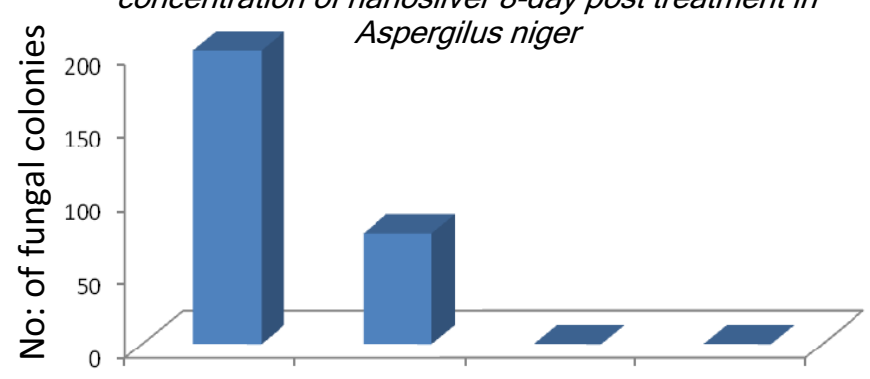

$$
\text { control Nanosilver Eucalyptus nanosilver \& Eucalyptus }
$$

Fig. 2. Comparison between fungi colony number of control and treatment groups in $12.5 \mathrm{ppm}$ concentration of nanosilver 24-day post treatment in Aspergilus niger

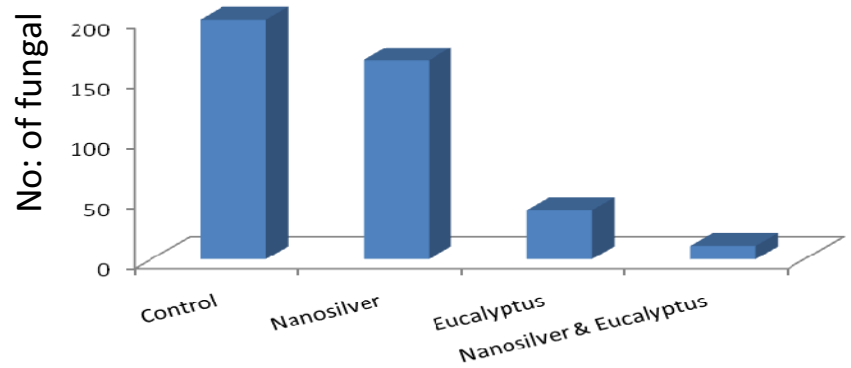

Fig. 3 Comparison between fungi colony number in $12.5 \mathrm{ppm}$ concentration of nanosilver different time post treatment in Aspergilus niger

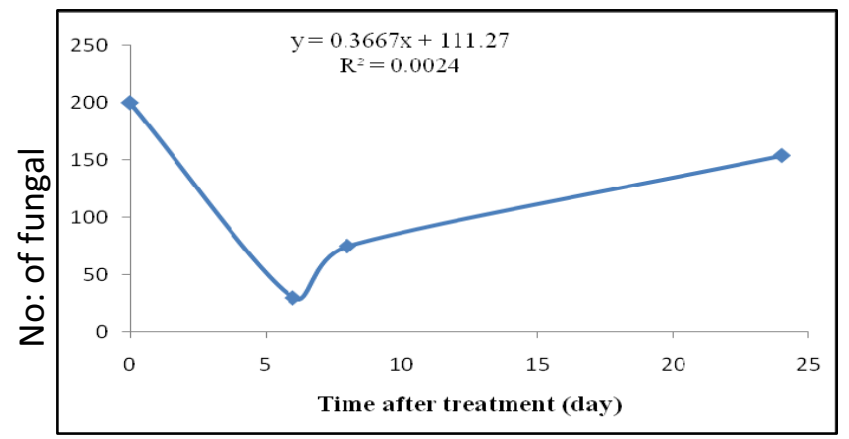

The inhibitory effect of nano-silver particles in $100 \mathrm{ppm}$ concentration on the diameter of fungi colonies showed meaningful reduction after 8 days of treatment $(P=0.00)$. The $150-\mathrm{ppm}$ concentration had the maximum inhibitory effect on fungi, so that no colony grew on the treated fungi. In addition, the minimum inhibitory concentration (MIC) for 100 spores was 37.5 and $18.25 \mathrm{ppm}$ for 24 and $48 \mathrm{hrs}$, respectively.

Conclusion

The inhibitory effect of nano-silver particles can be accounted for the diameter of silver ions. Due to their minute size, the silver ions have more contact surface with external space and consequently more effect on the cell membrane. On the other hand, the created molecular mechanism by nanosilver particles can be accounted free radicals of active oxygen. Therefore, silver oxidizes the oxygen atom producing oxygen ion, and it hydrolyzes the water producing $-\mathrm{OH}$, both productions are of active radicals and of the strongest antimicrobial factors.

The results showed that the silver nano-particles of 2.67 $\mathrm{nm}$ protected by hydrogel polymer chains present an excellent
Vol. 5 No. S3 (Mar 2012)

ISSN: 0974- 6846

antibacterial activity compared to the larger sized silver nanoparticles in the hybrid networks (Moudgil and Roberts, 2006). On the other hand, molecular mechanism provided by silver nano-particles can be attributed to production of free radicals. These free radicals were induced oxidative stress and program cell death in Aspergillus niger. These free radicals result in apoptosis, Programmed death of live cells, by attacking the intracellular organelles of fungi (Christoforidis et al., 2006). Some studies proved that the size and shape of the particle play central role in antimicrobial activity. The other mechanism is due to function of silver ions in colloidal solution. Converting bonds of $\mathrm{SH}$ to $\mathrm{S}-\mathrm{Ag}$ results in changes in microorganism.

In this mechanism, the nano-silver particles diffuse gradually silver ions. These ions substitute the $-\mathrm{SH}$ bonds to SAg ones in microorganism's membrane in a substitution reaction, resulting in microorganisms' death (Somayyeh et al., 2011). Of the important properties of nano-silver particles are very high effectiveness, non-allergic agent, with high durability against various conditions, compatibility with environment, resistance against heating, not increasing the microorganism's resistance and adaptation. Considering the antifungal effect of nano-silver particles in different size and shape, and because of no exact knowledge about their specific effect on Aspergillus niger, further researches are necessary for studying such effects. Considering the exact calculations of MIC of nano-silver particles in vitro, it is recommended to use this dosage for treating the fungal diseases in animals and then in humans. In addition, nano-silver particles can be proper alternatives for conventional antifungal agents. These found could be suggested that therapy of human fungal diseases in medicine will be performed with different size, shape, concentration, and combination of nano-particles.

\section{Reference}

1. Arikan S and Rex JH (2000) New agents for treatment of systemic fungal infections. Emerg. Drugs. 5, 135-160.

2. Arikan S, Lozano-Chiu M, Paetznick V and Rex JH ( 2001) In vitro susceptibility testing methods for caspofungin against Aspergillus and Fusarium isolates. Antimicrob Agents Chemother. 45, 327-330.

3. Christoforidis GA, Frank JA, Lindau M, Lockman, Geho DH, Jones CD, Petricoin EF, Liotta LA, Manninger SP, Qiang Y, Spence AM and Stupp SI. 2006) Nanoparticles: potential biomarker harvesters. Curr. Opin. Chem. Biol. 10, 56-61.

4. Moudgil BM and Roberts SM (2006) Designing a strategies for safety evaluation of nanomaterials. In: Nano-interface in a microfluidic chip to probe living VI. Characterization of nanoscale particles for cells: challenges and perspectives. Toxicol. Sci. 103, 6419-6424.

5. Nakagawa, Y, Shimazu K, Ebihara M and Nakagawa K (1999) Aspergillus niger pneumonia with fatal pulmonary oxalosis. J Infect Chemother. 5, 97-100.

6. Paux E, Tamasloukht M, Ladouce N, Sivadon P and GrimaPettenati J (2004) Identification of genes preferentially expressed during wood formation in Eucalyptus. Plant Mol. Biol. 55, 263-280.

7. Somayyeh M, Delavar HA and Motallebi A (2011) Toxicity Study of Nano-silver on Osteoblast Cancer Cell Line. Int. Nano Lett. 1,11-16.

8. Tahan C, Leung R, Zenner GM, Ellison KD, Crone WC and Miller CA (2006) Nanotechnology and improving packaged food quality and safety. Part 2: Nanocomposites. Am. J. Phys. 4, 443-448. 